\title{
Caracterización de los monómeros DGEBA y TMAB, y seguimiento de la reacción de entrecruzamiento por espectroscopia Raman
}

\author{
Characterization of DGEBA and TMAB monomers, and monitoring \\ the cross linking reaction by Raman spectroscopy
}

\author{
Fabio Augusto Mesa Rueda $\quad$ Alneira Cuéllar Burgos ${ }^{1} \quad$ Jairo Ernesto Perilla Perilla ${ }^{2}$ \\ Carlos Vargas-Hernández ${ }^{3}$
}

Recibido 6 de junio de 2012, aceptado 13 de junio de 2013

Received: June 6, 2012 Accepted: June 13, 2013

\begin{abstract}
RESUMEN
La espectroscopia Raman es una técnica ampliamente utilizada para el análisis físico-químico de materiales, ya que permite el estudio composicional y estructural de las muestras. Particularmente en los últimos años ha sido empleada para identificar los modos normales de vibración en la estructura química y en los grupos funcionales de monómeros y polímeros. Esta técnica dispersiva es la empleada en el estudio del sistema epoxi/amina donde se identifican los modos normales de vibración en el sistema compuesto por el oligómero epóxico diglicidil éter de bisfenol A (DGEBA) y la diamina trimetilen glicol di-p-aminobenzoato (TMAB). Se identificaron los principales modos normales de vibración de los grupos amino del TMAB y del anillo epóxico del DGEBA alrededor de 1626, 3333 y $3436 \mathrm{~cm}^{-1}$, y 736, 915, 933, 1131, 1249-1252, $1407,2713,2760$ y $3006 \mathrm{~cm}^{-1}$, respectivamente. Los picos asociados a los enlaces nitrógeno-hidrógeno simétricos y antisimétricos de la amina $\left(1626\right.$ y $\left.1687 \mathrm{~cm}^{-1}\right)$ y la vibración simétrica de los grupos $\mathrm{CH}_{2}$ del grupo epoxi $\left(3006 \mathrm{~cm}^{-1}\right)$ es un indicativo del avance de reacción de entrecruzamiento.
\end{abstract}

Palabras clave: Espectroscopia Raman, diglicidil éter de bisfenol A, DGEBA, trimetilen glicol di-paminobenzoato, TMAB, polímeros termofijos, mecanismo de curado.

\section{ABSTRACT}

Raman spectroscopy is a widely used technique for chemical and physical analyses of materials, which allows for the study of composition and the structure of the samples. Particularly in recent years, it has been used to identify the normal modes of vibration in the chemical structure and in the functional groups of monomers and polymers. This dispersive technique is employed in the study of the epoxy/amine system, where the normal modes of vibration in the system composed of the epoxy oligomer Diglycidyl ether of bisphenol-A (DGEBA) and trimethylene glycol di-p-aminobenzoate (TMAB) are identified. The principal normal modes of vibration were determined for amine groups of TMAB around 1626, 3333 y $3436 \mathrm{~cm}^{-1}$ and for epoxy rings of DGEBA around 736, 915, 933, 1131, 1249-1252, 1407, 2713, 2760 and 3006 $\mathrm{cm}^{-1}$. The peaks associated with nitrogen-hydrogen symmetric and antisymmetric bonds of the amine $\left(1626\right.$ y $\left.1687 \mathrm{~cm}^{-1}\right)$ and the symmetric vibration of the $\mathrm{CH}_{2}$ groups of the epoxy group $\left(3006 \mathrm{~cm}^{-1}\right)$ are indicative of the progress of crosslinking reaction.

Keywords: Raman spectroscopy, Diglycidyl ether of bisphenol-A, DGEBA, trimethylene glycol di-paminobenzoate, TMAB, thermosetting polymers, curing mechanism.

1 Universidad Nacional de Colombia. Laboratorio de Polímeros y Materiales Compuestos. Carrera 27 No 64-60. Manizales (Caldas), Colombia. E-mail: famesar@unal.edu.co; acuellarb@unal.edu.co

2 Universidad Nacional de Colombia. Departamento de Ingeniería Química y Ambiental. Carrera 30 No 45-03. Bogotá D.C., Colombia.E-mail: jeperillap@unal.edu.co

3 Universidad Nacional de Colombia. Laboratorio de Propiedades Ópticas de Materiales (POM). Carrera 27 N $^{\circ}$ 64-60. Manizales (Caldas), Colombia. E-mail: cvargash@unal.edu.co 


\section{INTRODUCCIÓN}

La espectroscopia Raman es una técnica vibracional ampliamente utilizada para el análisis físicoquímico de materiales, ya que permite el estudio no destructivo de las muestras. En los últimos 30 años esta técnica instrumental ha sido empleada para identificar los modos normales de vibración en la estructura química y los grupos funcionales de monómeros y polímeros. La espectroscopia Raman ha tenido un gran interés como técnica de caracterización, debido al avance de la tecnología de los láseres y los dispositivos optoelectrónicos de adquisición y procesamiento de señales, que han permitido su uso rutinario en el análisis químico y actualmente se emplea a nivel industrial en la caracterización y control de los productos. Tiene gran importancia en el estudio de sistemas epoxi/ amina para la producción de polímeros termofijos, ya que estos materiales se encuentran dentro de la clasificación dada a los materiales poliméricos, como respuesta a su comportamiento en función de la temperatura y se caracterizan estructuralmente por el elevado grado de reticulación o entrecruzamiento de las macromoléculas compuestas por unidades repetitivas con enlaces covalentes. Los polímeros termofijos tienden a degradarse ante un calentamiento progresivo sin llegar a ser líquidos [1]. Algunos polímeros que se encuentran en esta clasificación son las resinas epóxicas, fenólicas, poliéster insaturado, poliuretanos, acrilatos, urea-formaldehído, entre otras. Los sistemas epoxi/amina (EA) para la producción de resinas epóxicas entrecruzadas son materiales ampliamente usados como recubrimientos, adhesivos, matrices compuestas (con fibras sintéticas y/o naturales), entre otros, para la industria aeroespacial, transporte, electrónica, laminados, encapsulación, etc. [2]. Las redes de los polímeros termofijos, en particular las resinas epóxicas, se forman por reacción química entre monómeros, donde al menos uno de ellos tiene tres o más grupos reactivos por molécula (funcionalidad mayor o igual a tres) [3]. Dentro de los monómeros epóxicos comerciales (oligómeros epóxicos o resinas epóxicas) más usados se encuentran los de diglicidil éter de bisfenol A (DGEBA), diglicidil éter de bisfenol F (DGEBF), novolac, triglicidil para-aminofenol (TGAP), tetra diglicidil-4-4'-diamino difenil metano (TGDDM). Este tipo de monómeros puede reaccionar con más de 50 tipos de agentes de entrecruzamiento por un mecanismo catiónico o aniónico con el anillo epoxi (oxirano o $\alpha$-epoxi o 1,2-epoxi o epóxico) [2]. Algunos de los agentes de entrecruzamiento más usados son bases de Lewis, aminas primarias, aminas secundarias, aminas terciarias, amidas, ácidos orgánicos e inorgánicos, anhídridos, etcétera.

Específicamente se caracterizará el sistema conformado por un oligómero epóxico líquido tipo diglicidil éter de bisfenol A (DGEBA) y como agente de entrecruzamiento se usará la diamina aromática trimetilen glicol di-p-aminobenzoato (TMAB). El DGEBA es considerado industrialmente como un material de propósito general por sus aplicaciones en adhesivos de alto rendimiento, moldeo de piezas por vaciado, formulación de recubrimientos en carrocerías de automóviles, formulación de recubrimientos para envases, recubrimiento de estructuras en ambientes corrosivos (p.ej. medio ambiente marino o químico), formulación de pinturas por fotocurado, etc. [4]. Por otra parte, el TMAB es usado como agente de curado en resinas epóxicas y en elastómeros de poliuretano, y provee en la formulación excelentes propiedades mecánicas y químicas, estabilidad hidrolítica, buen desempeño en altas temperaturas y suministra resistencia química a hidrocarburos, solventes, contaminantes y ozono; además de ser un producto industrialmente seguro aprobado por la FDA en los Estados Unidos [5]. La importancia del seguimiento de la reacción de entrecruzamiento es debida a que las propiedades físico-químicas de los materiales son dependientes del grado de conversión.

La caracterización estructural local de estos monómeros se realizó por la técnica de espectroscopia Raman, que permite identificar los modos normales de vibración e inferir su estructura química y sus respectivos grupos funcionales que intervendrán en la reacción de curado. La técnica Raman es una técnica de alta resolución y permite discriminar los modos normales de vibración de forma más definida que otras técnicas espectroscópicas, debido a que es una técnica de dispersión y no de absorción. No necesitan de una preparación previa de las muestras en estudio, y una de las principales ventajas de la espectroscopia Raman es la utilización de luz visible, con lo que la óptica a utilizar en el microscopio es relativamente simple, junto con una más alta resolución espacial. 


\section{SISTEMA REACTIVO EPOXI/DIAMINA (EA) UTILIZADO}

Para el análisis del sistema EA se escogió la resina epóxica líquida $D E R \circledast$-331 tipo DGEBA de la casa Dow Chemical Inc. suministrada por la empresa Andercol S.A. (Medellín, Colombia). La verificación del peso equivalente epoxi se realizó utilizando la norma ASTM D1652 [6], obteniendo un valor promedio de $190,77 \mathrm{~g} / \mathrm{eq}$ que se encuentra dentro del intervalo ofrecido por el proveedor.

Con este valor de equivalente epoxi se puede determinar la cantidad de agente de entrecruzamiento para una relación de grupos epoxi a grupos amínicos estipulados en la formulación del polímero termofijo. Algunas propiedades y la estructura de este oligómero se muestran en la Tabla 1 y Figura 1, respectivamente.

Tabla 1. Propiedades del oligómero epóxico.

\begin{tabular}{|l|c|}
\hline Peso Equivalente Epoxi (hoja técnica): & $182-192 \mathrm{~g} / \mathrm{eq}$. \\
\hline Peso Equivalente Epoxi (ASTM D1652): & $190,77 \mathrm{~g} / \mathrm{eq}$. \\
\hline Porcentaje Epóxido (hoja técnica): & $22,4-23,6 \%$ \\
\hline Porcentaje Epóxido (ASTM D1652): & $22,54 \%$ \\
\hline Viscosidad @ $25^{\circ} \mathrm{C}(\mathrm{mPa} \cdot \mathrm{s}):$ & $11000-14000$ \\
\hline Densidad @ $25^{\circ} \mathrm{C}:$ & $1,16 \mathrm{~g} / \mathrm{ml}$ \\
\hline
\end{tabular}

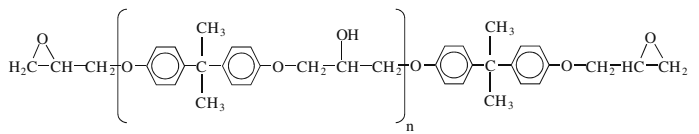

Figura 1. Estructura química del oligómero epóxico.

El agente de entrecruzamiento TMAB es una diamina aromática de Air Products and Chemicals Inc. y se conoce con el nombre comercial de Versalink®740M. Algunas propiedades y la estructura del monómero se muestran en la Tabla 2 y la Figura 2, respectivamente.

Tabla 2. Propiedades de la diamina TMAB.

\begin{tabular}{|l|c|}
\hline Peso Eq. de Hidrógenos amínicos: & $78,5 \mathrm{~g} / \mathrm{eq}$. \\
\hline Punto de Fusión: & $125^{\circ} \mathrm{C}$ \\
\hline Densidad @ $140^{\circ} \mathrm{C}:$ & $1,14 \mathrm{~g} / \mathrm{ml}$ \\
\hline
\end{tabular}<smiles>Nc1ccc(C(=O)OCCOC(=O)c2ccc(N)cc2)cc1</smiles>

Figura 2. Estructura química del agente de curado.
La Figura 3 presenta el mecanismo simplificado de reacción en tres etapas entre un equivalente epoxi y un hidrógeno amínico para un sistema EA. En la primera y segunda etapa ocurre la apertura de uno de los anillos epóxicos por un ataque nucleofílico sobre el carbón terminal del anillo oxirano, con la conversión de este en un grupo hidroxílico [7-8]. Un grupo amínico primario puede reaccionar sucesivamente con dos grupos oxirano debido a los dos hidrógenos activos de la amina. Hay que tener en cuenta que la resina epóxica es bifuncional y el agente de entrecruzamiento tiene dos grupos amino con cuatro equivalentes hidrógeno, y por lo tanto el mecanismo descrito anteriormente es similar para el otro grupo oxirano y amínico en los monómeros y genera una red con enlaces covalentes característico de un polímero termofijo.

En la tercera etapa, estos grupos hidroxílicos pueden intervenir formando una reacción de eterificación con otro grupo epóxico debido a temperaturas altas, conversiones altas y por el efecto competitivo entre aminas secundarias de gran impedimento estérico con los grupos epóxicos. Además, la apertura del anillo epóxico se puede catalizar por los compuestos formados que contengan grupos hidroxílicos [7].

(1)

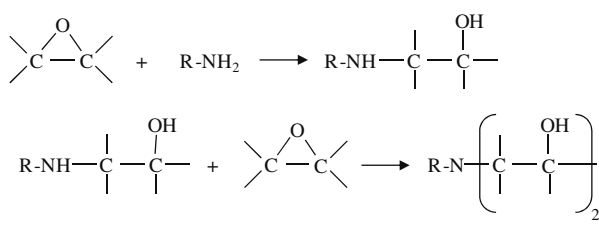

(3)

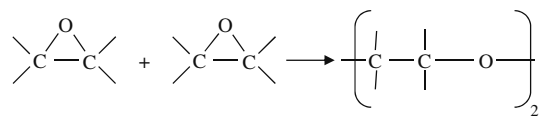

Figura 3. Mecanismo de reacción simplificado entre aminas y grupos epóxicos.

En la formulación del sistema EA se tomó una relación 1:1 de equivalentes de grupos epoxi a hidrógenos amínicos. Para el análisis por espectroscopia Raman del polímero termofijo se entrecruzó el sistema EA a $150^{\circ} \mathrm{C}$ en un horno de convección forzada. Las Figuras 4 y 5 muestran los resultados de calorimetría diferencial de barrido (DSC) usando un equipo TA Instruments Q-2000 con un flujo de $\mathrm{N}_{2}$ de $50 \mathrm{~cm}^{3} /$ min para determinar el comportamiento del calor de reacción a $150^{\circ} \mathrm{C}$ y el tiempo necesario para regresar a la línea base. 


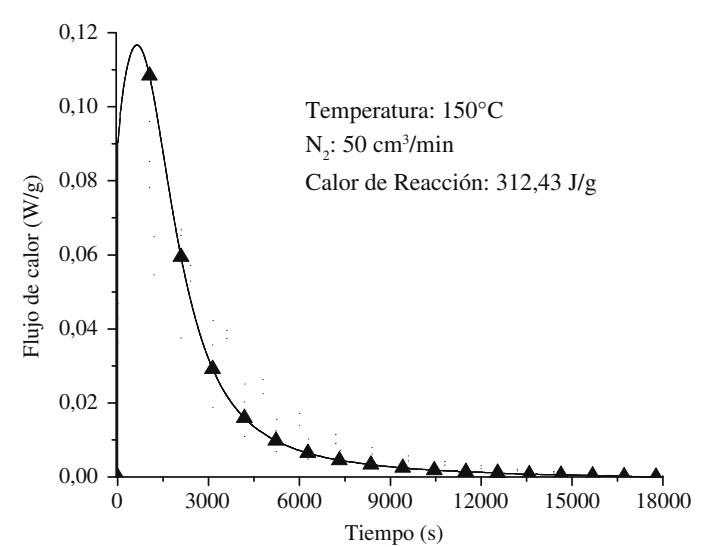

Figura 4. Calor de reacción para el sistema EA a temperatura constante $\left(150^{\circ} \mathrm{C}\right)$.

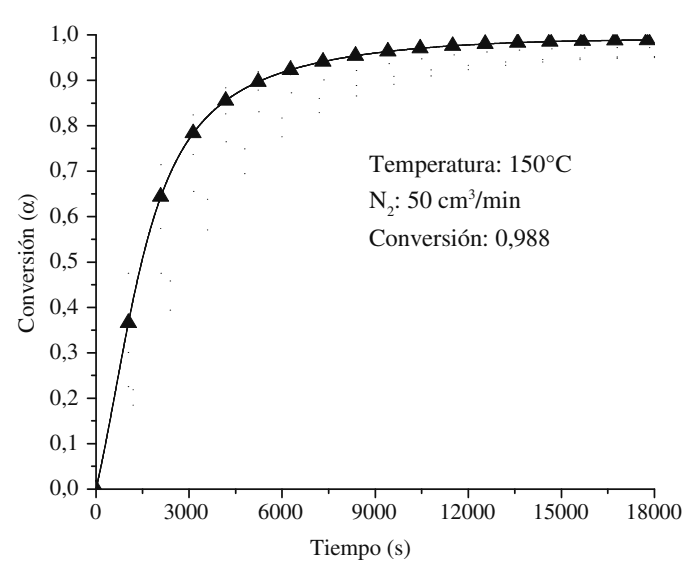

Figura 5. Conversión del sistema EA a temperatura constante $\left(150^{\circ} \mathrm{C}\right)$.

De este análisis de calorimetría diferencial de barrido se obtuvo que el tiempo de reacción, el calor de reacción y la conversión máxima fueron 300 minutos (18000 s), 312,43 J/g y 0,988, respectivamente.

\section{CARACTERIZACIÓN POR ESPECTROSCOPIA RAMAN}

La caracterización de los monómeros TMAB y DGEBA y del material termofijo se hizo a través de espectroscopia confocal Raman de alta resolución, utilizando un equipo LabRamHR-800 (Horiba Jobin-Yvon), con un láser de excitación de longitud de onda $473 \mathrm{~nm}$, potencia nominal $10 \mathrm{~mW}$ y un objetivo 10X.

Dentro de los grupos funcionales de mayor importancia en este sistema EA y en el polímero termofijo que deben ser identificados se encuentran el anillo epóxico para el DGEBA y las aminas primarias en el TMAB. Las Figuras 6 y 7 muestran los espectros Raman para la diamina TMAB en los intervalos de 500-2000 y 2000-4000 $\mathrm{cm}^{-1}$, respectivamente.

Teniendo en cuenta información reportada por varios autores para otras aminas y para compuestos con grupos estructurales similares, se identificaron los modos normales de vibración del monómero TMAB. La Tabla 3 describe cada uno de los modos vibracionales más importantes para los espectros Raman obtenidos en las Figuras 6 y 7 , y se comparan con los reportados en la literatura [9-13].

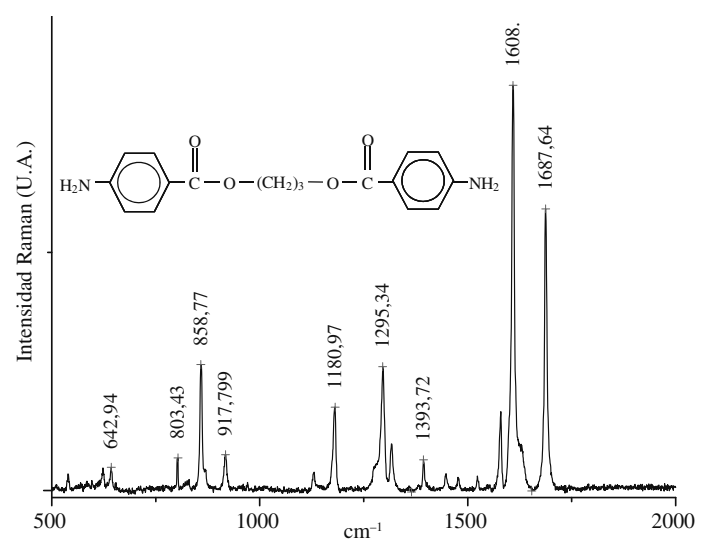

Figura 6. Espectro Raman para la diamina TMAB. Intervalo de $500-2000 \mathrm{~cm}^{-1}$.

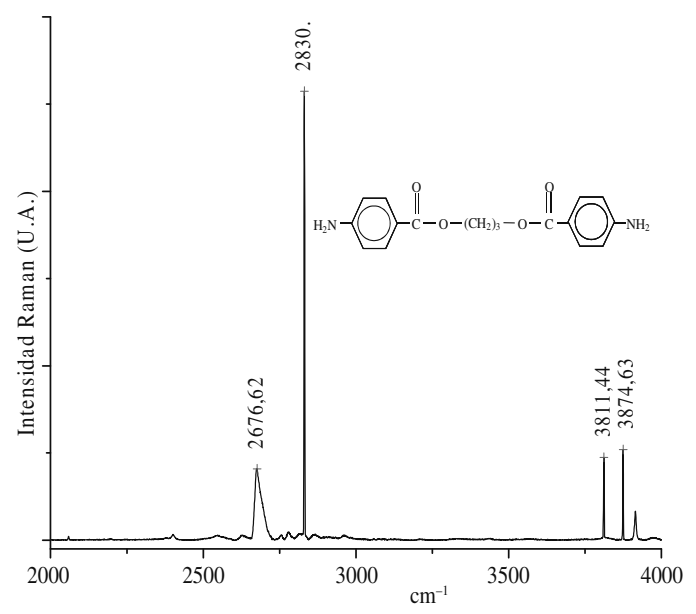

Figura 7. Espectro Raman para diamina TMAB. Intervalo $2000-4000 \mathrm{~cm}^{-1}$. 
Tabla 3. Comparación e identificación con la literatura y con este trabajo de las frecuencias Raman para la diamina TMAB.

\begin{tabular}{|c|c|c|c|c|c|}
\hline & & & \\
\hline Modo vibracional & $\begin{array}{l}\text { Literatura } \\
\quad\left(\mathrm{cm}^{-1}\right)\end{array}$ & $\begin{array}{c}\text { Este } \\
\text { trabajo } \\
\left(\mathrm{cm}^{-1}\right)\end{array}$ & \multirow{2}{*}{$\begin{array}{l}\mathrm{v}(\mathrm{N}-\mathrm{H}) \text { Vibración de enlaces } \\
\text { nitrógeno-hidrógeno en los } \\
\text { grupos amínicos (simétricos } \\
\text { y asimétricos). Aminas } \\
\text { primarias y secundarias. }\end{array}$} & \multirow{2}{*}{$\begin{array}{l}3330-3400, \\
3300-3600, \\
3304-3366, \\
3340-3361, \\
3404-3422, \\
3367,3454\end{array}$} & \multirow[t]{2}{*}{$\begin{array}{l}3333(w) \\
3436(w)\end{array}$} \\
\hline $\begin{array}{l}\mathrm{v}(H) \text { Vibración de los enlaces } \\
\text { de hidrógeno en el plano del }\end{array}$ & $\begin{array}{l}622(\mathrm{~m}) \\
622(\mathrm{~m})\end{array}$ & $623(\mathrm{~m})$ & & & \\
\hline
\end{tabular}

w: Weak; b) m: Medium; c) s: Strong; d) vs: Very Strong.

Las Figuras 8 a 10 representan los modos normales de vibración para cada uno de los picos más representativos de la diamina TMAB en el intervalo de 500 a $2000 \mathrm{~cm}^{-1}$ y de 2000 a $4000 \mathrm{~cm}^{-1}$.

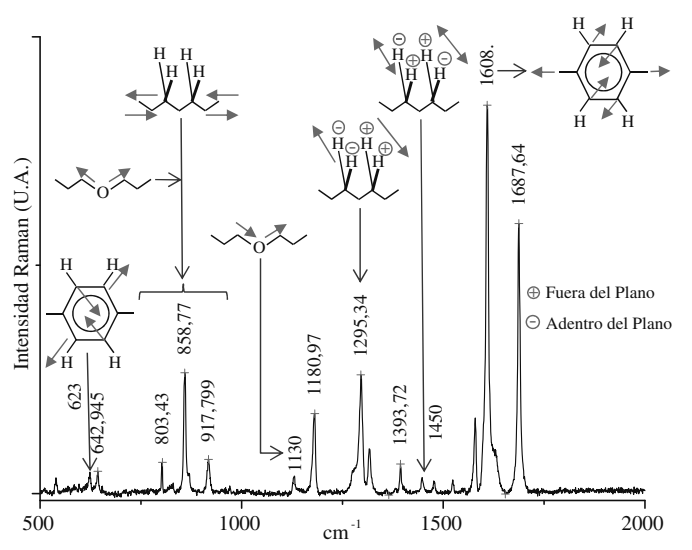

Figura 8. Representación de los modos normales de vibración para la diamina TMAB en el intervalo de $500-2000 \mathrm{~cm}^{-1}$.

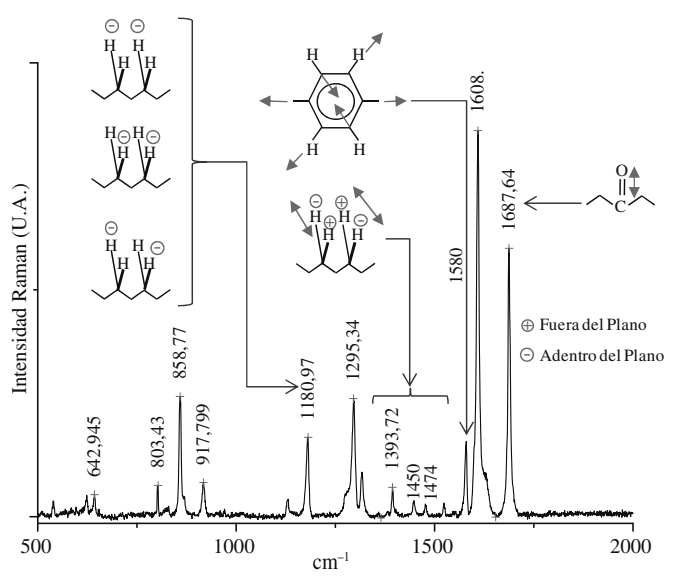

Figura 9. Representación de los modos normales de vibración para la diamina TMAB en el intervalo de $500-2000 \mathrm{~cm}^{-1}$. 


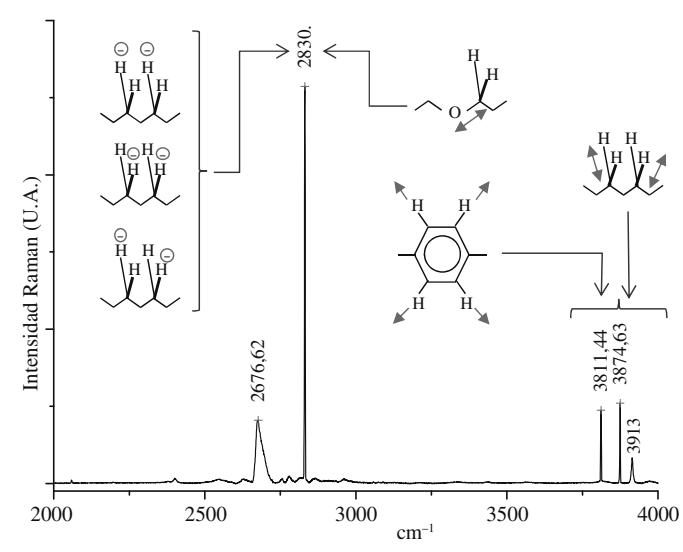

Figura 10. Representación de los modos normales de vibración para la diamina TMAB en el intervalo de $2000-4000 \mathrm{~cm}^{-1}$.

Para el agente de entrecruzamiento, los picos correspondientes al grupo amínico son importantes para el seguimiento de la reacción de entrecruzamiento, pero en este caso no fue posible debido a su intensidad débil comparada con otros grupos. Esto se debe al efecto de apantallamiento que tiene otros modos normales de vibración sobre los grupos amino. Por lo tanto, es conveniente estudiar los espectros Raman en las regiones de 3300-3500 $\mathrm{cm}^{-1}$ y en $1626 \mathrm{~cm}^{-1}$, ya que la disminución de la intensidad de estos picos es un indicativo del avance de reacción de entrecruzamiento debido a la conversión.

La gráfica inserta en la Figura 11 muestra el espectro Raman en la zona de $3300-3500 \mathrm{~cm}^{-1}$, donde se aprecian dos picos que representan los modos

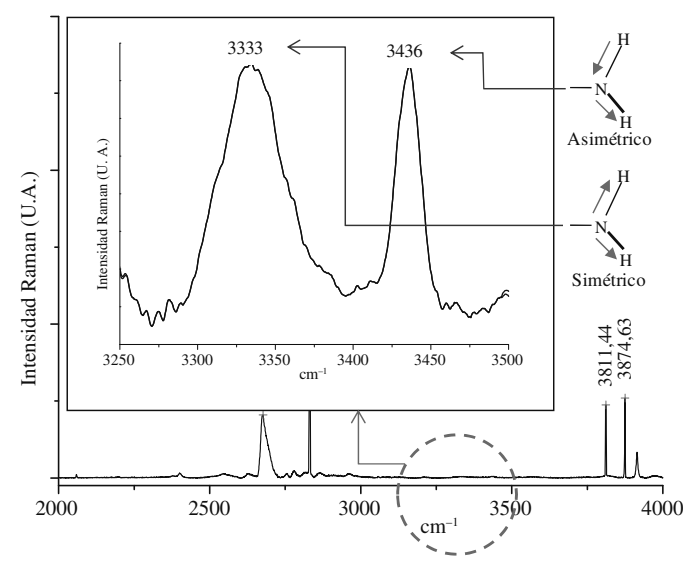

Figura 11. Representación de los modos normales de vibración para los grupos amínicos. normales de vibración de los enlaces v(N-H) simétrico $\left(3333 \mathrm{~cm}^{-1}\right)$ y asimétrico $\left(3436 \mathrm{~cm}^{-1}\right)$. Es claro que comparándolos con otros picos característicos de la amina, la intensidad de estos modos vibracionales es débil y pueden llegar a ser de difícil seguimiento cuando no se tiene una apropiada resolución.

Con un procedimiento similar al realizado con la amina y con información reportada para resinas epóxicas de compuestos con grupos funcionales y estructurales similares, se hizo la respectiva identificación para el $D G E B A$. La Tabla 4 identifica los picos más importantes que se presentan en la Figura 12. Estos resultados son comparados con los reportados en la literatura [10-18].

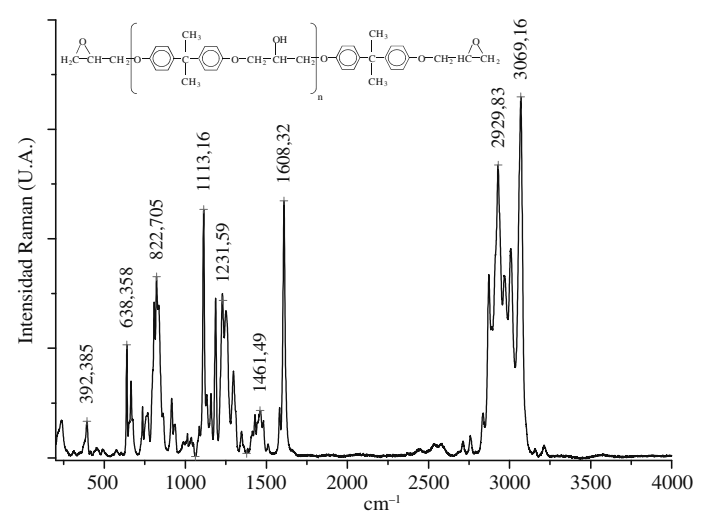

Figura 12. Espectro Raman para la resina DGEBA en el intervalo de $150-4000 \mathrm{~cm}^{-1}$.

Tabla 4. Comparación e identificación de frecuencias Raman para el oligómero epóxico DGEBA.

\begin{tabular}{|c|c|c|}
\hline Modo vibracional & $\begin{array}{l}\text { Literatura } \\
\left(\mathrm{cm}^{-1}\right)\end{array}$ & $\begin{array}{l}\text { Este trabajo } \\
\left(\mathrm{cm}^{-1}\right)\end{array}$ \\
\hline $\begin{array}{l}\mathrm{v}(C-H) \text { Deformación } \\
\text { de los enlaces carbono- } \\
\text { hidrógeno fuera del } \\
\text { plano del anillo } \\
\text { aromático }\end{array}$ & $\begin{array}{l}641(\mathrm{~s}) \\
667(\mathrm{~m}) \\
640(\mathrm{~m})\end{array}$ & $\begin{array}{l}638(\mathrm{~m}) \\
666(\mathrm{~m})\end{array}$ \\
\hline $\begin{array}{l}\mathrm{v}(C-C) \text { Vibración de } \\
\text { los enlaces carbono- } \\
\text { carbono contiguo al } \\
\text { anillo epóxico }\end{array}$ & $\begin{array}{l}736(\mathrm{~s}) \\
762(\mathrm{~m})\end{array}$ & $\begin{array}{l}736(\mathrm{~m}) \\
762(\mathrm{~m})\end{array}$ \\
\hline $\begin{array}{l}\mathrm{V}\left(\mathrm{CH}_{2}\right) \text { Deformación } \\
\text { adentro del plano del } \\
\text { grupo } \mathrm{CH}_{2} \text { en el anillo } \\
\text { epóxico }\end{array}$ & $\begin{array}{l}846(\mathrm{~m}) \\
840-853\end{array}$ & $837(\mathrm{~m})$ \\
\hline $\begin{array}{l}\mathrm{v}(C-O-C) \text { Vibración del } \\
\text { anillo epóxico }\end{array}$ & $\begin{array}{c}908(\mathrm{~m}) \\
924(\mathrm{~s}) \\
912-920 \\
914(\mathrm{~m}) \\
1211(\mathrm{~s})\end{array}$ & $\begin{array}{l}915(\mathrm{~m}) \\
933(\mathrm{~m}) \\
1228(\mathrm{~s})\end{array}$ \\
\hline
\end{tabular}




\begin{tabular}{|c|c|c|}
\hline Modo vibracional & $\begin{array}{c}\text { Literatura } \\
\left(\mathrm{cm}^{-1}\right)\end{array}$ & $\begin{array}{c}\text { Este trabajo } \\
\left(\mathrm{cm}^{-1}\right)\end{array}$ \\
\hline $\begin{array}{l}\mathrm{v}(C-) \text { Vibración del } \\
\text { enlace sustituido en el } \\
\text { anillo aromático. }\end{array}$ & $\begin{array}{l}1010(w) \\
1036(w)\end{array}$ & $\begin{array}{l}1011(w) \\
1035(w)\end{array}$ \\
\hline $\begin{array}{l}\mathrm{v}(C-H) \text { Deformación } \\
\text { adentro del plano de } \\
\text { los enlaces carbono- } \\
\text { hidrógeno en el anillo } \\
\text { aromático }\end{array}$ & $1010(w)$ & 1011(w) \\
\hline $\begin{array}{l}\mathrm{v}(C-H) \text { Vibración de } \\
\text { los enlaces carbono- } \\
\text { hidrógeno en el } \\
\text { anillo aromático y } \\
\text { deformaciones dentro } \\
\text { del plano. }\end{array}$ & 1113(s) & 1113(s) \\
\hline $\begin{array}{l}\mathrm{v}\left(\mathrm{CH}_{2}\right) \text { Deformación } \\
\text { de los enlaces carbono- } \\
\text { hidrógeno en el anillo } \\
\text { epóxico (wagging) }\end{array}$ & $1130(\mathrm{~m})$ & $1131(\mathrm{~m})$ \\
\hline $\begin{array}{l}\mathrm{v}\left(\mathrm{C}-\mathrm{CH}_{3}\right) \text { Vibración de } \\
\text { los enlaces carbono- } \\
\text { carbono (del grupo } \\
\text { metilo) y deformación } \\
\text { de los grupos metilo } \\
\text { adentro del plano. }\end{array}$ & $1186(\mathrm{~s})$ & 1186(s) \\
\hline $\begin{array}{l}\mathrm{v}(C-H) \text { Deformación } \\
\text { adentro del plano de } \\
\text { los enlaces carbono- } \\
\text { hidrógeno en el anillo } \\
\text { aromático }\end{array}$ & $1190(s)$ & $1186(\mathrm{~s})$ \\
\hline $\begin{array}{l}\mathrm{v}(\mathrm{C}-\mathrm{O}) \text { Vibración del } \\
\text { enlace carbono-oxígeno } \\
\text { con el grupo fenilo y } \\
\text { con el carbono del } \\
\text { grupo } \mathrm{CH}_{2} .\end{array}$ & $1232(\mathrm{~s})$ & $1231(\mathrm{~s})$ \\
\hline $\begin{array}{l}\mathrm{v}(\mathrm{C}-\mathrm{O}) \text { Vibración del } \\
\text { enlace carbono-oxígeno } \\
\text { con el carbono del } \\
\text { grupo } \mathrm{CH}_{2} .\end{array}$ & $\begin{array}{l}1248(s) \\
1256(s)\end{array}$ & $1249(\mathrm{~s})$ \\
\hline $\begin{array}{l}\mathrm{V}(C-O-C) \text { Deformación } \\
\text { dentro del plano del } \\
\text { anillo epóxico. }\end{array}$ & $\begin{array}{c}1259(\mathrm{~s}) \\
1265(\mathrm{~m}) \\
1270(\mathrm{~m}) \\
\end{array}$ & $1249-1252$ \\
\hline $\begin{array}{l}\mathrm{V}\left(\mathrm{CH}_{2}\right) \text { Deformación } \\
\text { de los enlaces carbono- } \\
\text { hidrógeno en el anillo } \\
\text { epóxico (twisting) }\end{array}$ & $1407(\mathrm{~m})$ & $1407(w)$ \\
\hline $\begin{array}{l}\mathrm{v}(\mathrm{C}-\mathrm{H}) \text { Deformación } \\
\text { de los enlaces carbono- } \\
\text { hidrógeno adentro y } \\
\text { afuera del plano en los } \\
\text { grupos } \mathrm{CH}_{2}\end{array}$ & $\begin{array}{l}1450(\mathrm{~m}) \\
1464(\mathrm{~m}) \\
1477(\mathrm{w})\end{array}$ & $\begin{array}{l}1450(\mathrm{~m}) \\
1461(\mathrm{~m}) \\
1481(\mathrm{w})\end{array}$ \\
\hline $\begin{array}{l}\mathrm{v}(C C) \text { Vibración de } \\
\text { los enlaces carbono- } \\
\text { carbono dentro y } \\
\text { fuera del plano anillo } \\
\text { aromático, deformación } \\
\text { de los enlaces CH }\end{array}$ & $\begin{array}{c}1584(\mathrm{~m}) \\
1583(\mathrm{~m}) \\
1604(\mathrm{~m}) \\
1602(\mathrm{~s}) \\
1612(\mathrm{~m}) \\
1614(\mathrm{~s}) \\
\end{array}$ & $\begin{array}{l}1581(\mathrm{~m}) \\
1608(\mathrm{~m})\end{array}$ \\
\hline
\end{tabular}

\begin{tabular}{|c|c|c|}
\hline Modo vibracional & $\begin{array}{l}\text { Literatura } \\
\left(\mathrm{cm}^{-1}\right)\end{array}$ & $\begin{array}{c}\text { Este trabajo } \\
\left(\mathrm{cm}^{-1}\right)\end{array}$ \\
\hline $\begin{array}{l}\mathrm{v}(-\mathrm{CH}-) \text { Vibración } \\
\text { simétrica de los enlaces } \\
\text { en el grupo } \mathrm{CH} \text { del } \\
\text { anillo oxirano }\end{array}$ & $2716(w)$ & 2713(w) \\
\hline $\begin{array}{l}\text { v(-CH-) Vibración } \\
\text { asimétrica de los } \\
\text { enlaces en el grupo } \\
\mathrm{CH} \text { del anillo oxirano }\end{array}$ & 2763(w) & $2760(w)$ \\
\hline $\begin{array}{l}\left.\text { v(-O-CH} 2^{-}\right) \text {Vibración } \\
\text { del grupo } \mathrm{CH}_{2} \text { en el éter. }\end{array}$ & $\begin{array}{c}2833-2838 \\
2840(\mathrm{~m}) \\
\end{array}$ & $2836(\mathrm{~m})$ \\
\hline $\begin{array}{l}\mathrm{v}\left(-\mathrm{O}-\mathrm{CH}_{2}-\right) \text { Vibración } \\
\text { simétrica del grupo } \mathrm{CH}_{2} \\
\text { en el éter. }\end{array}$ & $\begin{array}{c}2873(\mathrm{~m}) \\
2875(\mathrm{~m}) \\
2873-2875\end{array}$ & $2873(\mathrm{~m})$ \\
\hline $\begin{array}{l}\mathrm{v}\left(-\mathrm{O}-\mathrm{CH}_{2^{-}}\right) \text {Vibración } \\
\text { asimétrica del grupo } \\
\mathrm{CH}_{2} \text { en el éter. }\end{array}$ & $\begin{array}{c}2931(\mathrm{~m}) \\
2922-2925 \\
2919(\mathrm{~m})\end{array}$ & $2929(\mathrm{~m})$ \\
\hline $\begin{array}{l}\mathrm{v}(C-H) \text { Vibración } \\
\text { asimétrica del enlace } \\
\text { carbono-hidrógeno en } \\
\text { los grupos metilo }\end{array}$ & $\begin{array}{c}2971(\mathrm{~m}) \\
2944-2953\end{array}$ & $2970(\mathrm{~m})$ \\
\hline $\begin{array}{l}\mathrm{v}\left(-\mathrm{CH}_{2^{-}}\right) \text {Vibración } \\
\text { simétrica de los grupos } \\
\mathrm{CH}_{2} \text { en los anillos } \\
\text { oxirano }\end{array}$ & $\begin{array}{c}3004(\mathrm{~m}) \\
3010(\mathrm{~m}) \\
3002-3005 \\
3000(\mathrm{~m})\end{array}$ & $3006(\mathrm{~m})$ \\
\hline $\begin{array}{l}\mathrm{v}(C-H) \text { Vibración de } \\
\text { los enlaces carbono- } \\
\text { hidrógeno en el } \\
\text { anillo aromático con } \\
\text { deformación adentro } \\
\text { y afuera del plano }\end{array}$ & $\begin{array}{c}3055(\mathrm{~s}) \\
3062(\mathrm{~s}) \\
3069(\mathrm{~s}) \\
3070(\mathrm{~s}) \\
3038-3046 \\
3068-3073 \\
3071(\mathrm{~s})\end{array}$ & $3069(\mathrm{~s})$ \\
\hline
\end{tabular}

w: Weak; b) m: Medium; c) s: Strong; d) vs: Very Strong.

De acuerdo al mecanismo de reacción mostrado en la Figura 3, el seguimiento a los grupos oxirano sirve para conocer el alcance del entrecruzamiento. Hay varios picos asociados a los modos normales de vibración de los anillos epoxi, algunos de ellos tienen mayor intensidad que otros y la disminución con la conversión de cualquiera de ellos es indicativo del avance en la reacción de entrecruzamiento. Los picos que se pueden considerar como potenciales candidatos para el seguimiento del curado con base en diferentes modos vibracionales del grupo epoxi son $736,915,933,1131,1249-1252,1407,2713$, 2760 y $3006 \mathrm{~cm}^{-1}$. Las Figuras 13 a 17 representan los modos normales de vibración representativos del DGEBA en los intervalos de 200 a $1800 \mathrm{~cm}^{-1}$ y 2400 a $3600 \mathrm{~cm}^{-1}$. 


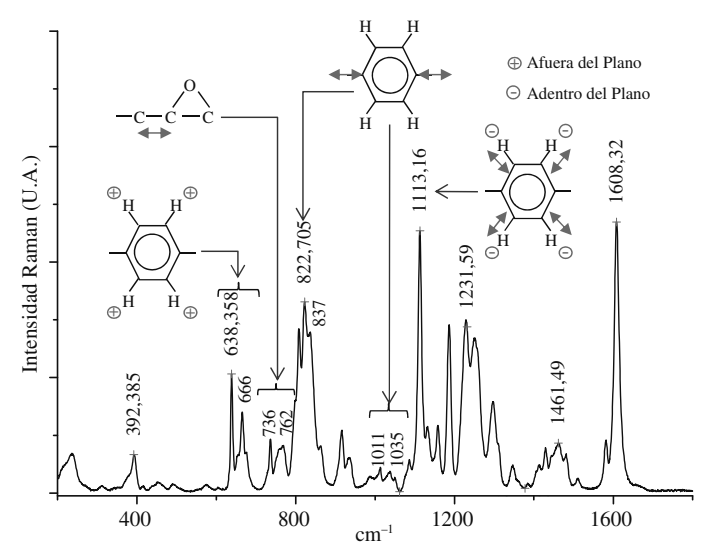

Figura 13. Representación de los modos normales de vibración para la resina DGEBA en el intervalo de $200-1800 \mathrm{~cm}^{-1}$.

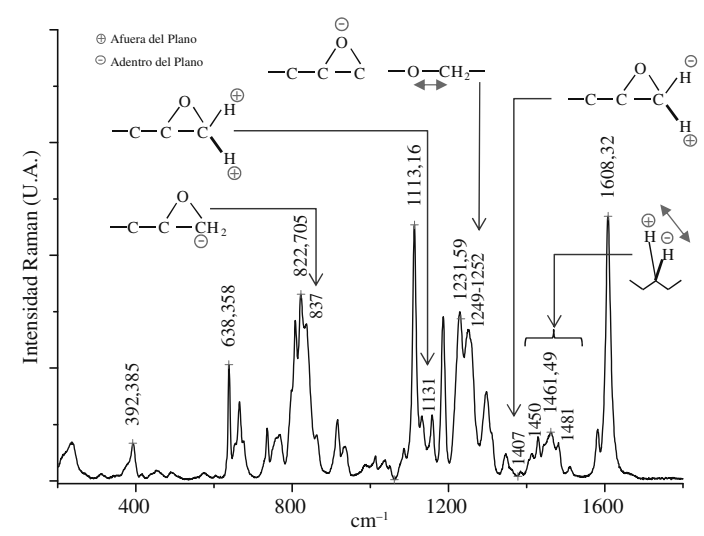

Figura 14. Representación de los modos normales de vibración para la resina DGEBA en el intervalo de $200-1800 \mathrm{~cm}^{-1}$.

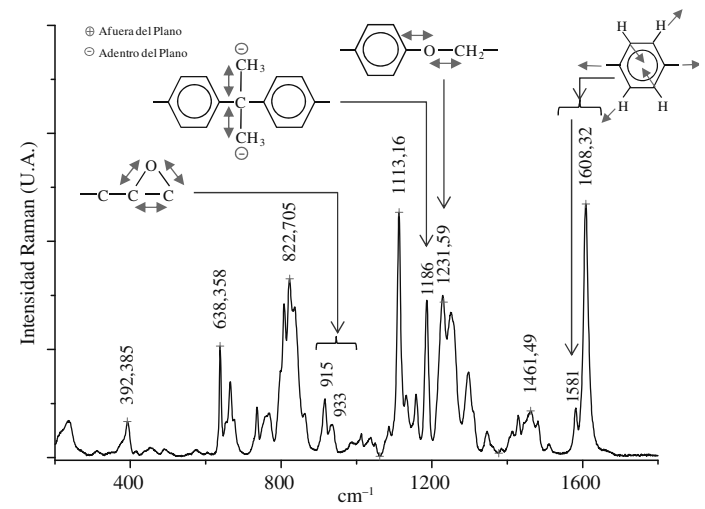

Figura 15. Representación de los modos normales de vibración para la resina DGEBA en el intervalo de $200-1800 \mathrm{~cm}^{-1}$.

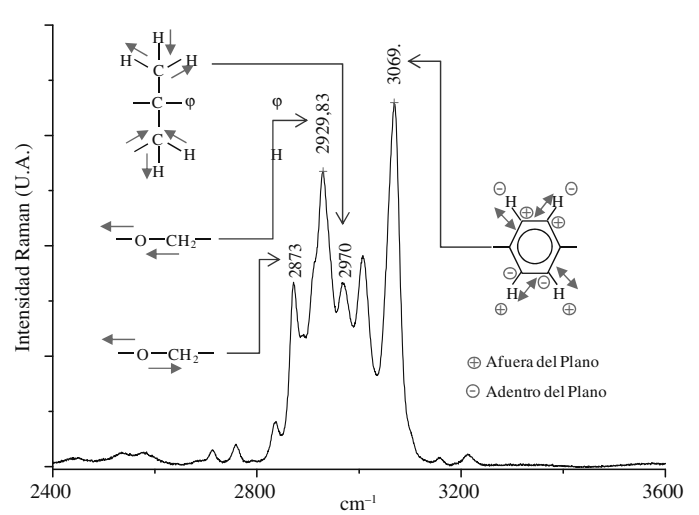

Figura 16. Representación de los modos normales de vibración para la resina DGEBA en el intervalo de $2400-3600 \mathrm{~cm}^{-1}$.

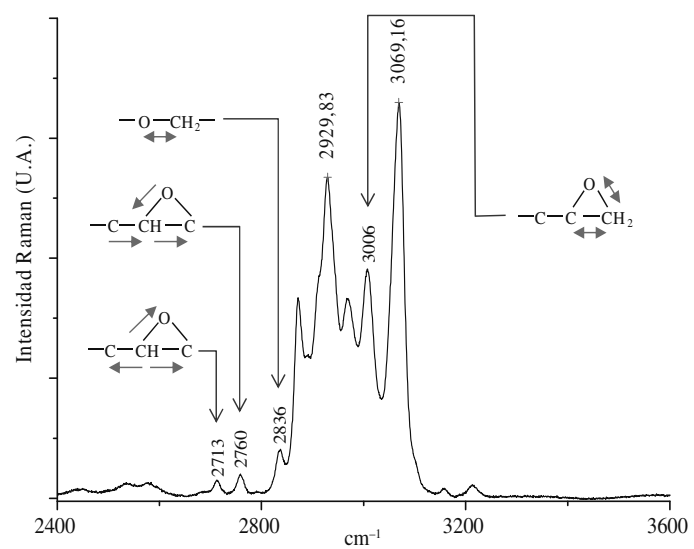

Figura 17. Modos normales de vibración para la resina DGEBA en el intervalo de 2400$3600 \mathrm{~cm}^{-1}$.

La Figura 18 muestra el cambio en el comportamiento de los picos representativos de la diamina TMAB, la resina epóxica DGEBA y el polímero entrecruzado a $150^{\circ} \mathrm{C}$ durante 300 minutos. Para los modos normales vibracionales de los grupos amínicos: a) enlaces nitrógeno-hidrógeno simétricos y asimétricos (1626 $\left.\mathrm{cm}^{-1}\right)$; b) enlaces nitrógeno-hidrógeno simétrico $\left(3333 \mathrm{~cm}^{-1}\right)$ y c) enlaces nitrógeno-hidrógeno asimétrico $\left(3436 \mathrm{~cm}^{-1}\right)$. Por otra parte, el cambio en los picos representativos de los anillos epoxi se pueden observar para los siguientes modos vibracionales: a) enlaces carbono-carbono contiguo al anillo epóxico $\left(736 \mathrm{~cm}^{-1}\right)$; b) deformación de los enlaces carbono-hidrógeno en el anillo epóxico $\left(1131 \mathrm{~cm}^{-1}\right)$; c) deformación dentro del plano del anillo epóxico $\left(1249 \mathrm{~cm}^{-1}\right)$; d) vibración simétrica de los enlaces en el grupo $\mathrm{CH}$ del anillo oxirano 


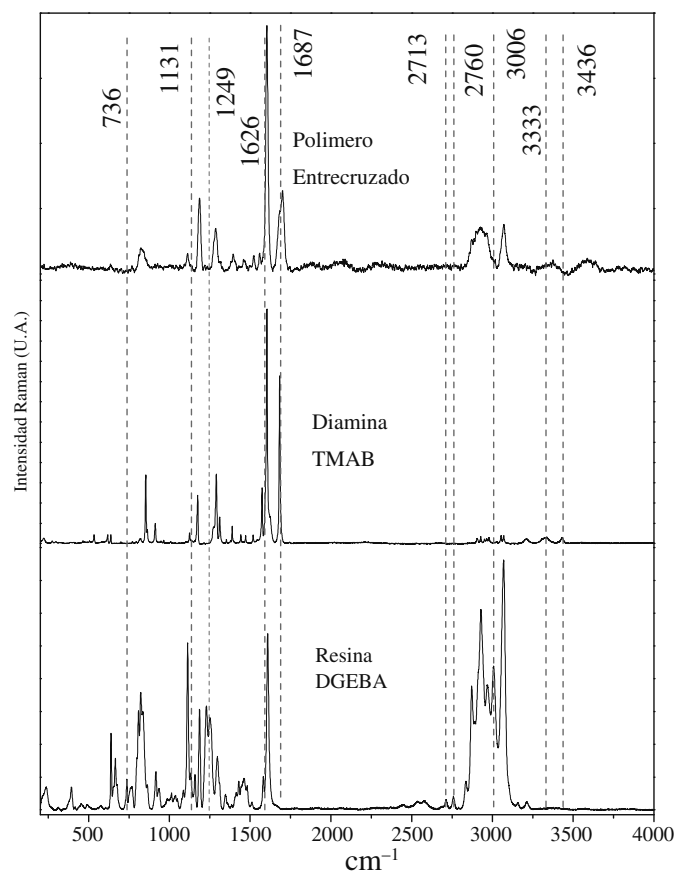

Figura 18. Comparación de los picos representativos de los grupos amino y epoxi de la diamina TMAB, la resina DGEBA y el polímero termofijo.

$\left(2713 \mathrm{~cm}^{-1}\right)$; e) vibración asimétrica de los enlaces en el grupo $\mathrm{CH}$ del anillo oxirano $\left(2760 \mathrm{~cm}^{-1}\right)$ y f) vibración simétrica de los grupos $\mathrm{CH}_{2}$ en los anillos oxirano $\left(3006 \mathrm{~cm}^{-1}\right)$.

Como picos de control y como indicativo del grado de conversión se han tomado los picos ubicados alrededor de 1626, 2713 y 3006 cm$^{-1}$ y 1626 y 1687 $\mathrm{cm}^{-1}$, correspondientes a la resina DGEBA y diamina TMAB, que están relacionados con la interacción entre el anillo oxirano y los grupos amino. Las intensidades normalizadas $I_{N}^{T M A B}, I_{N}^{D G E B A}$ y $I_{N}^{\text {Poli }}$ son las áreas bajo la curva de la suma de los picos en los espectros Raman para la resina DGEBA, diamina TMAB y el polímero entrecruzado, respectivamente. Los cuales se relacionan mediante las ecuaciones:

$I_{N}^{T M A B}=\frac{\sum_{i}^{n} I_{i}^{T M A B}}{I_{\text {Total }}^{T M A B}}, I_{N}^{D G E B A}=\frac{\sum_{i}^{n} I_{i}^{\text {DGEBA }}}{I_{\text {Total }}^{\text {DGEBA }}}, I_{N}^{\text {Poli }}=\frac{\sum_{i}^{n} I_{i}^{\text {Poli }}}{I_{\text {Total }}^{\text {Poli }}}$

$$
I_{N}^{c}=\frac{I_{N, 1687}^{\text {Poli }}}{I_{N, 1687}^{T M A B}}
$$

Donde, $I_{N}^{c}, I_{N, 1687}^{\text {Poli }}$ y $I_{N, 1687}^{T M A B}$ son el porcentaje de conversión, La intensidad normalizada correspondiente al polímero entrecruzado y la intensidad normalizada de la diamina TMAB, todos asociados al pico de $1687 \mathrm{~cm}^{-1}$. Los resultados arrojan un valor de 0,94 , indicando que mediante esta metodología de la espectroscopia Raman los valores están en concordancia relativa con lo obtenido por calorimetría diferencial (ver Figura 5).

\section{CONCLUSIONES}

La espectroscopia Raman es una técnica de alta eficiencia para identificar los modos vibracionales de la estructura e identificación de grupos funcionales en el sistema EA con los monómeros DGEBA y TMAB. Con esta metodología se considera que posiblemente el seguimiento de la reacción de entrecruzamiento del sistema DGEBA/TMAB se manifiesta a partir de la dinámica de los picos 1626, 3333 y $3436 \mathrm{~cm}^{-1}$ para los grupos amino del TMAB, y 736, 915, 933, 1131, $1249-1252,1407,2713,2760$ y $3006 \mathrm{~cm}^{-1}$ para el anillo epóxico del DGEBA. En cualquiera de los casos, la intensidad de los picos disminuye con el avance de la reacción de entrecruzamiento. Los resultados relativos obtenidos de conversión del sistema EA por espectroscopia Raman concuerdan con los registrados por calorimetría diferencial de barrido.

En próximos trabajos se desea mostrar el uso de la espectroscopia Raman para estudiar la dinámica de los modos vibracionales representativos en un proceso de entrecruzamiento del sistema EA y comparar los resultados con calorimetría diferencial de barrido para hacer el seguimiento de cinéticas de polimerización.

\section{AGRADECIMIENTOS}

Los Autores agradecen al Laboratorio de Polímeros y Materiales Compuestos de la Universidad Nacional de Colombia sede Manizales. El presente trabajo ha sido apoyado parcialmente por el proyecto Semilleros de Investigación de la Dirección de Investigaciones (DIMA) de la Universidad Nacional de Colombia sede Manizales.

\section{REFERENCIAS}

[1] G. Odian. "Principles of Polymerization". John Wiley \& Sons. 2004. 
[2] H. Lee. "Handbook of Epoxy Resins". McGraw-Hill. 1967.

[3] J.P. Pascault, H. Sautereau, J. Verdu and R.J.J. Williams. "Thermosetting Polymers". Marcel Dekker. 2002.

[4] Dow Chemical Company. "Epoxy Resin: Selection Guide". Catalog. 1998.

[5] Air Products and Chemicals Inc. "Versalink®740M Diamine Curative For High-Performance Polyurethane Elastomers and Epoxies". Catalog. 2004.

[6] ASTM International. "ASTM D 1652-97: Standard Test Methods for Epoxy Content of Epoxy Resins". Catalog. 1997.

[7] F.A. Mesa, A. Cuellar, C. Vargas-Hernández and J.E. Perilla Perilla. "Cinética y mecanismo de entrecruzamiento de diglicidil éter de bisfenol-A con trimetilen glicol di-paminobenzoato". Dyna. Vol. 77, Issue 162, pp. 21-29. 2010.

[8] E. Girard-Reydet, C.C. Riccardi, H. Sautereau and J.P. Pascault. "Epoxy -Aromatic Diamine Kinetics. 1. Modeling and Influence of the Diamine Structure". Macromolecules. Vol. 28, pp. 7599-7607. 1995.

[9] Horiba Jobin Yvon Companies. "Raman data and Analysis: Raman Spectroscopy for Analysis and Monitoring". Catalog. 2007.

[10] P. Musto, M. Abbate, G. Ragosta and G. Scarinzi. "A study by Raman, near-infrared and dynamic-mechanical spectroscopies on the curing behaviour, molecular structure and viscoelastic properties of epoxy/anhydride networks". Polymer. Vol. 48, pp. 3703-3716. 2007.

[11] R. Nyquist. "Interpreting infrared, raman, and nuclear magnetic resonance spectra". Academic Press. 2001.
[12] E. Overbeke, J. Devaux, R. Legras, J.T. Carter, P.T. McGrail and V. Carlier. "Raman Spectroscopy and DSC Determination of Conversion in DDS-Cured Epoxy Resin: Application to Epoxy-Copolyethersulfone Blends”. Applied Spectroscopy”. Vol. 55, pp. 540-551. 2001.

[13] B. Riegel, W. Kiefer, S. Hofacker and G. Schottner. "FT-Raman Spectroscopic Investigations on the Organic Crosslinking in Hybrid Polymers. Part I: Model Reactions of Simple Epoxides". Applied Spectroscopy. Vol. 54, pp. 1384-1390. 2000.

[14] J.F. Aust, K.S. Booksh and M.L. Myrick. "Novel In Situ Probe for Monitoring Polymer Curing". Applied Spectroscopy. Vol. 50, pp. 382-387. 1996.

[15] K.E. Chike, M.L. Myrick, R.E. Lyon and S.M. Angel. "Raman and Near-Infrared Studies of an Epoxy Resin". Applied Spectroscopy. Vol. 47, pp. 1631-1635. 1993.

[16] T.M. Don and J.P. Bell. "Fourier transform infrared analysis of polycarbonate/epoxy mixtures cured with an aromatic amine". Journal of Applied Polymer Science. Vol. 69, pp. 2395-2407. 1998.

[17] S. Farquharson, J. Carignan, V. Khitrov, A. Senador and M. Shaw. "Development of a Phase Diagram to Control Composite Manufacturing using Raman Spectroscopy", Personal Communication. 2007.

[18] B. Riegel, W. Kiefer, S. Hofacker and G. Schottner. "FT-Raman Spectroscopic Investigations on the Organic Crosslinking in Hybrid Polymers Part II: Reactions of Epoxy Silanes". Journal of Sol-Gel Science and Technology. Vol. 24, pp. 139-145. 2002. 